\title{
MODELING RISKS IN REAL ESTATE DEVELOPMENT PROJECTS: A CASE FOR EGYPT
}

\author{
Mohamed MARZOUK ${ }^{1, *}$, Ahmed ABOUSHADY ${ }^{1}$ \\ ${ }^{1}$ Structural Engineering Department, Faculty of Engineering, Cairo University, 12613 Giza, Egypt
}

Received 18 August 2016; accepted 4 October 2017

\begin{abstract}
Risk analysis is a vital step in the succession of construction projects. However, no adequate researches have been conducted to assess, and quantify risk events in real estate projects in developing countries, and particularly in Egypt.This research recommends Fuzzy Quantitative Risk Assessment Model to quantify risk factors participated in real estate development projects. Model is composed of two components: 1) Fuzzy Fault Tree (FT) that determines root causes of each risk, probability of its occurrence, and probability of mitigation strategies failure; and 2) Fuzzy Event Tree (ET) that calculates crisp value of Expected Monetary Value (EMV) of allowance of mitigation of the identified risks. Causes of risk are determined through literature review and interviews with experts in field. Risk probability occurrence is determined using five linguistic terms, defined either triangular or trapezoidal membership functions which are developed using modified horizontal approach and an interpolation technique. Two-step Delphi technique is used to achieve consensus on the root causes and logical representation of the Fault Tree. Fuzzy importance analysis is performed to rank different root causes for identified risks according to their criticality to probability of occurrence. A Case Study is presented to evaluate results obtained from model, in terms of Expected Monetary Value (EMV), and fuzzy probability of failure for each risk participated in case study.
\end{abstract}

Keywords: risk management, fuzzy sets, fault tree, event tree, real estate development projects, mitigation strategy, Egypt.

Supplementary material associated with this article can be found, in the online version, at https://doi.org/10.3846/ijspm.2018.6270

\section{Introduction}

Risk management can be described as an essential process in establishment of real estate construction projects, which may have an impact on construction industry as a whole. Real estate industry plays a crucial role in raising national income and solving social problems in both developed and developing countries. In 2011, it contributed with almost 5\% to US Gross Domestic Product (GDP). This figure is less the peak of 2006, which is $8.9 \%$ of the GDP (Approximately $\$ 1.195$ trillion). Real estate construction industry is labour intensive. As such, any decline in residential construction contributes to recession's high unemployment rate (Amadeo, 2012). Moreover, it plays an essential role in flourishing economies of developing countries. In a developing country like Egypt, the real estate construction industry contributes to the Egyptian Government Budget with 2.83 percent from the Egyptian national income (Alexandria Bank Economic Research, 2012). Moreover, real estate projects differ from other types of construction projects because they are considered the riskiest construction project since they require from developer to pay large amount of capital in the form of purchasing land. Also, they are considered as long-term investment project. The owner profit will be achieved at the end of the projects when units are sold. Therefore, real estate projects are characterized as negative cash flow project because of owner continuous payment. Due to high levels of risk and uncertainty associated with the Real Estate Construction Industry, project teams in real estate projects need to assess risk events encountered in their projects in order to eliminate vagueness, imprecision, and unavailable data and information. Moreover, when risk events are expected, project team members compute the price of real estate projects at different scenarios. This can be done using the expected monetary value (EMV) which provides project teams with a method to calculate the cost of the project if one risk event or many risk events occur. Also, due to current political and economic hard situations facing these projects after Egyptian Revolution in 2011 affected real estate construction industry immensely. Thus, due to in existence of adequate risk analysis in real estate

*Corresponding author. E-mail: mm_marzouk@yahoo.com 
construction projects in Egypt, economic difficulties that encountered in real estate development projects after 2011 revolution, and the instability in markets prices due to increase in the cost of purchasing equipment, materials, and continuous devaluation of local currency against foreign currency, the real estate project teams want to evaluate risks in projects to produce a prioritized list of risks, which help them addressing vagueness and imprecision that they are currently facing. The assessment methodology can be changed and applied to different types of construction projects by changing risk events, and expert judgment.

\section{Literature review}

PMI (2008) defined project risk management cycle as "the cycle of identifying, qualifying, quantifying, and planning response risks." Different researches have tackled qualitative risk assessment. KarimiAzari, Mousavi, Mousavi, and Hosseini (2011) have analysed risk models using matrix method to select construction projects. Moreover, Foong and Nordin (2010) used Risk Assessment Matrix to assist engineers in reducing safety, environmental, and economic impacts of risk hazards. Also, Markowski and Mannan (2008) studied Fuzzy Risk Matrix. The research described a methodology for development of fuzzy matrix to decrease the number of accidents using different scenarios. Another study, was performed by Robert (2004) that described a 3-dimensional approach to qualify risks, where first axis represents possibility of occurrence of risk, second axis represents possibility of consequence, and third axis represents level of consequence. On the opposite side, in literature, different methods have been applied to quantify risks events. Kangari and Riggs (1989) organized methods into two main groups: the first is classical models (Probability models); and the second is subjective models, such as fuzzy set method. Quantitative analysis for risk management problem contains determining certain available failure of the system, and consequence to decrease failure probabilities of system components and loses, respectively (Kangari \& Riggs, 1989). Selecting optional decision alternatives is developed to reduce risk events or expected loss (Al-Bahar \& Crandall, 1990). Several techniques have been adopted to perform quantitative analysis including; decision trees, neural networks, Monte Carlo Simulation, and fuzzy logic.

Levner, Ganoulis, Linlov, and Benayahu (2007) studied multi objective assessment of risks for artificial marine system using fuzzy decision trees estimation. However, it is should be mentioned here that decision trees are incapable of computing accurate probability values unless historical data exist, which is not available in our case at hand. Further, they neither provide a methodology for detecting critical risk events nor define a clear sequence for root causes identification associated with risk events. Maria-Sanchez (2005) quantified risk events in terms of costs in construction projects using neural networks. The most common risks in infrastructure projects were identified and analysed to evaluate risk impacts to contractor's profit. Al-Sobiei, Arditi, and Polat (2005) designed a model to predict contractors' default risks in Saudi Arabia using artificial neural network (ANN) and genetic algorithm techniques. Angelini, Giacomo, and Roli (2008) studied credit risk evaluation using neural network approach. In spite of fact that neural networks need historical data, neural network lack ability to explain logic behind how model is applied to produce outputs. Further, neural networks lack ability to include mitigation strategies in risk assessment. On the other hand, Monte Carlo Simulation was either integrated with fuzzy logic to study risk assessment in construction (Sadeghi, Fayek, \& Pedrycz, 2010) or used to compute the consequence of a cash flow hazards on project success (Javid \& Seneviratne, 2000). Nevertheless, Monte Carlo Simulation lacks the ability to identify causes of risk events. Also, the results obtained from Monte Carlo Simulation are wide in range.

Fuzzy logic had been first recommended by Zadeh (1965). Subsequently, a lot of research efforts have been carried using fuzzy logic. A. Chan, D. Chan, and Yeung (2009) presented a model describing "Fuzzy Techniques" in Construction Management Assessment. Markowski, Mannan, and Bigoszewska (2009) proposed a methodology to implement fuzzy risk matrix that is suitable for safety analysis. Shaheen, Fayek, and AbouRizk (2007) developed model to estimate range in cost. It is worth noting that Fuzzy-Logic method cannot provide a clear methodology for computing the Expected Monetary Value of a risk event. Further, effect of development of mitigation strategies is not considered by the Fuzzy-Logic method, which could be better dealt with using fault and event tree analysis. NASA (2002) provided a definition for FT as "a graphical diagram of different parallel and successive combinations of faults that may lead to undesired event existence". FT has been applied in different applications including; Construction Dispute Negotiation Failure (Yiu, Cheung, \& Lok, 2015), prediction of oil and gas pipeline failure probability (Yuhua \& Datao, 2005), reliability analysis of solar array (Wu, Yan, \& Xie, 2011), and recognition of nuclear power plant systems failure (Abdelhai, 1993).

Tyagi, Pandey, and Kumar (2011) studied fuzzy FT analysis for fault diagnosis in power transformer using beta distribution. Abdelgawad and Fayek (2011) examined quantitative risk events using fuzzy fault tree analysis. They used subjective terms to analyse risks probability of occurrence. Their proposed approach offers the risk coordinators the ability to rank events according to probability of risk event. Song, Zhang, and Chan (2009) proposed a fuzzy FT assessment based on Takagi and Sugeno model with application to navigate the system.

Real estate project developers face difficulties in evaluating risks associated with their projects. They indeed need to compute bidding price and EMV of each risk to accommodate impacts of risk. This article proposes fuzzy quantitative risk analysis model, which combines fuzzy FT and ET assessment to analyse risks in real estate 
development projects. Model assists real estate developers in identifying critical causes of risk events and conducting fuzzy importance analysis on fault trees. Also, it assists in determining the actual cost of mitigation strategy if risk event occurs by computing EMV of each risk, using ET analysis. Model improves over previous quantitative risk management models by computing the actual cost of the mitigation strategy of each risk event.

\section{Research methodology}

The methodology of developing the fuzzy quantitative risk analysis model is depicted in Figure 1. These stages are described in later sub-sections.

\begin{tabular}{|c|}
\hline $\begin{array}{l}\text { Data collection } \\
\text { - Collect root } \\
\text { - Establish linguistic terms to assess probability }\end{array}$ \\
\hline $\begin{array}{l}\text { Conduct qualitative risk analysis using fault tree } \\
\text { - Develop Boolean matrix } \\
\text { - Develop working Boolean matrix } \\
\text { - Conduct analysis }\end{array}$ \\
\hline
\end{tabular}

\section{$\sqrt{1}$}

Conduct quantitative risk analysis using fault tree

- Represent each linguistic term using $\alpha$-cuts

- Represent the top event fuzzy probability using minimum cut set

- Conduct fuzzy arithmetic operations

- Defuzzify the top event using the mean of maximum

- Conduct fuzzy importance analysis

Conduct fuzzy fault tree analysis using mitigation
strategy
- Compute fuzzy probability of mitigation strategies
Conduct fuzzy importance analysis to select the
most suitable mitigation strategy

Figure 1. Methodology and model development

\subsection{Data collection}

This stage is divided into two steps; collect root causes for each critical risk event existed in Real Estate Development Projects, and establish linguistic terms of these critical risk events. Collect root causes step is concerned with defining the root causes for each critical risk event and mitigation strategies failure using various techniques, such as interviews, Delphi, brain storming and check list. In this model, literature review and interviews with fifteen experts in real estate development projects were conducted using indirect method. The two-step Delphi technique was used to achieve consensus among experts. Establish linguistic term step is concerned with assessing probability of occurrence of critical risks. Fuzzy linguistic terms are defined using interviews with fifteen experts. Experts have previous experience of more than twenty years in both real estate development projects, and risk assessment. Experts agreed to have five-point scale which range from Very Low $(V L)$ to Very High $(V H)$ in order to compute probability of occurrence for each risk. Experts were then asked to develop membership function for each linguistic term. Modified horizontal approach with interpolation technique was used to develop membership function (MF). Process of developing MF of input and output variables determines shapes of MF for both input and output variables using modified horizontal approach (Hauptmanns, 1988; Kangari \& Riggs, 1989; Khan \& Abbasi, 1999; Javid \& Seneviratne, 2000; Maria-Sanchez, 2005; Levner et al., 2007; Markowski, Mannan, \& Bigoszewska, 2009; Marsh \& Fayek, 2010, Elbarkouky \& Fayek, 2011). Detailed process of constructing membership functions using modified horizontal approach with interpolation is explained in Aboushady (2012).

\subsection{Conducting qualitative fault tree analysis}

This stage assesses basic events fuzzy probability of occurrence resulted from Stage 1, and identifies the minimal cut sets (MCS). Ayyub (2013) defined MCS as "MCS with condition that basic event non-occurrence from this set may result in top event non-occurrence." To illustrate computation of the MCS using Hauptmanns' (1988) algorithm, Figure 3 illustrates the FT structure that captures the failure of financial system as a top event (TE). TE is connected with three gate events using OR gate which are; increase in interest rate (GE1), increase in Taxes (GE2), and inflation (GE3). Each gate event is connected with a list of basic events. The first stage in qualitative FT analysis to convert FT structure into Boolean matrix abbreviated as (BM), which is composed of 0 and 1 to represent no connection or existing connection, respectively. Table S1 (supplementary material) lists Boolean Matrix (BM) for illustrative example of FT shown in Figure 2.

The second step involves creating empty matrix known as Working Boolean Matrix abbreviated as (WBM), and begin assessment from top event. Third step contains replacing TE in WBM with basic events $(\mathrm{BE})$ and gate events (GE), known as Connection List (CL). 


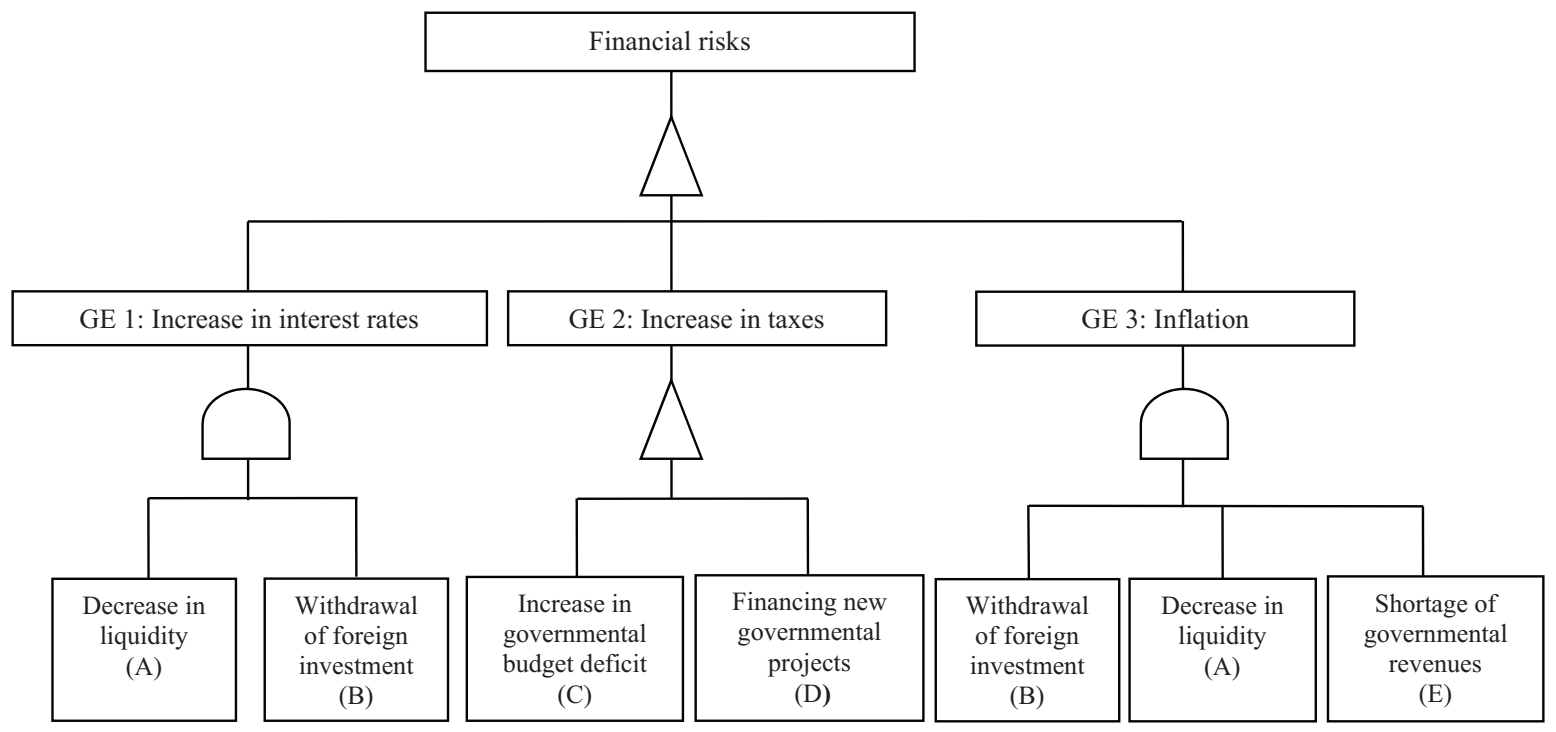

Figure 2. Illustrative example of qualitative FT structure

Relation between BE and TE using OR and AND gates were determined through interviews with fifteen experts, using the indirect method. The consensus among experts was achieved using two-step Delphi Technique. Table S2 (supplementary material) summarizes the steps to conduct the Qualitative FT Analysis.

Fourth step is to go through rows of the WBM to examine connectivity. For instance, Table 2 first row is read as " $\mathrm{C}, \mathrm{D}$ ". $\mathrm{BE}$ in a row are connected to another row using Union operator " $U$ ". As such, top event is represented using Equation 1 which is simplified using Equation 2.

$$
\begin{aligned}
& \mathrm{TE}=(\mathrm{A} \cap \mathrm{B} \cap \mathrm{C}) \mathrm{U}(\mathrm{A} \cap \mathrm{B}) \mathrm{U}(\mathrm{C} \cup \mathrm{D}) ; \\
& \mathrm{TE}=\mathrm{E} \cap(\mathrm{A} \cap \mathrm{B}) \mathrm{U}(\mathrm{C} \cup \mathrm{D}) .
\end{aligned}
$$

\subsection{Conducting quantitative assessment fault tree}

Main aim of performing quantitative FT assessment is to compute TE fuzzy probability of occurrence. This is achieved through assessing BE fuzzy probability of occurrence linguistically by choosing one of linguistic variables presented in Figure 2. The main steps for performing fuzzy quantitative fault tree analysis are:

1) Use alpha-cut ( $\propto$-cut) principle to represent the linguistic variable chosen.

2) Use minimal cut set identified in fuzzy qualitative fault tree to describe fuzzy probability of top event.

3) Perform fuzzy arithmetic operations to convert the " $U$ " and " $\cap$ " in the minimal cut set equations (MCS).

Verma, Srividya, and Gaonkar (2007) procedure is adopted which is reported by Abdelgawad (2011) to perform analysis. The $\propto$-cut of fuzzy probability of events connected by an " $U$ " gate is defined using Equation 3 for mutually exclusive events. Whereas, fuzzy probability of events connected by an " $\cap$ " is represented by Equation 4 .

$$
\begin{aligned}
& \operatorname{FP}(\text { Top event })^{\propto}= \\
& \left.\left\{1-\prod_{i=1}^{n}\left[1-\left(a_{i}+\left(b_{i}-a_{i}\right)\right)^{\propto}\right], 1-\prod_{i=1}^{n}\left(d_{i}-\left(d_{i}-c_{i}\right)\right)^{\propto}\right]\right\} \\
& \left\{1-\prod_{i=1}^{n}\left[1-\left(a_{i}+\left(b_{i}-a_{i}\right)\right)^{\propto}\right], 1-\prod_{i=1}^{n}\left[1-\left(d_{i}-\left(d_{i}-c_{i}\right)\right)^{\propto}\right]\right\},
\end{aligned}
$$

where: $n$ is No. of events linked with " $U$ "; $a$ stands for minimum value; $b$ and $c$ stand for most likely value; $d$ stands for maximum value of MF.

$$
\operatorname{FP}(\text { Top event })^{\propto}=\left\{\begin{array}{l}
\prod_{i=1}^{s}\left[a_{i}+\left(b_{i}-a_{i}\right)^{\propto}\right], \\
\prod_{i=1}^{s}\left[d_{i}-\left(d_{i}-c_{i}\right)^{\propto}\right]
\end{array}\right\} .
$$

It should be noted that $S$ is number of events connected by " $\cap$ ".

TE fuzzy probability is defuzzified using mean of maximum (MOM) method where MF at $\propto$ equals 1 represents most confident level. Purpose of calculation of Fuzzy Probability of TE is to determine the percentage of existence of TE if TE occurs. Subsequently, fuzzy importance analysis is performed to identify critical causes using Equation 5 as per Khan and Abbasi (1999). Fuzzy Probability of TE computed as value of lower bound at $\propto$ equals 1 plus value of the upper bound at $\propto$ equals 1 divided by 2 . Then, qualitative and quantitative $\mathrm{FT}_{\mathrm{S}}$ are analysed to suggest most suitable mitigation strategies.

$$
\mathrm{FIM}=\frac{T E_{1}-T E_{2}}{T E_{1}} \cdot 100 \%,
$$

where: $\mathrm{TE}_{1}$ is the top event fuzzy probability, assuming that all root causes will happen; $\mathrm{TE}_{2}$ is the top event fuzzy probability, assuming each root cause is eliminated in turn. 


\subsection{Analyze mitigation strategies}

Each identified mitigation strategy is then analysed by considering the failure of mitigation to be the top event, and repeating steps for stages of data collection, qualitative and quantitative fuzzy fault tree (Abdelgawad, 2011). For example, let us suggest that the real estate projects teams have identified two mitigation strategies: Mitigation Strategy 1 named "Encourage Local and Foreign Investment" and Mitigation Strategy 2 named "Increase the Governmental Revenues". Project team members can investigate each mitigation strategy to detect its causes that leads to failure of mitigation strategies. Figure 3 illustrates fault tree to represent mitigation strategies failure, and fuzzy probability of BE.

Qualitative FT analysis is conducted as described earlier. Table S3 (supplementary material) illustrates the Working Boolean Matrix of the failure of Mitigation Strategies 1 and 2. Subsequently, the minimal cut set equations are developed by converting each row's connection with its related basic event as well as connects basic events with each row using intersection operator " $\cap$ ". $B E$ in a row are connected to $\mathrm{BE}$ in another row using union operator " $U$ ". By conducting this step to all rows in Table 3, the failure of Mitigation Strategy 1 can be represented using Equation 6. Whereas, the failure of Mitigation Strategy 2 can be represented using Equation 7.

$$
\begin{aligned}
& \mathrm{FM}_{1}=\mathrm{A} \cup \mathrm{B} ; \\
& \mathrm{FM}_{2}=\mathrm{C} \cup \mathrm{D} \cup \mathrm{E} .
\end{aligned}
$$

After conducting qualitative FT analysis, quantitative FT analysis is performed using steps defined in the quantitative FT analysis section. Fuzzy probability of failure of $F_{M 1}$ is represented by Equation 8 .

$$
\left.\mathrm{F}_{\mathrm{P}}(F M 1)^{\infty}=\left[\left(1-\mathrm{F}_{\mathrm{P}}(A)^{\propto}\right) \cdot\left(1-\mathrm{F}_{\mathrm{P}}(B)^{\propto}\right)\right)\right],
$$

where: $\mathrm{F}_{\mathrm{P}}(A)^{\infty}, \& \mathrm{~F}_{\mathrm{P}}(B)^{\infty}$ are fuzzy probability of $\mathrm{BE}$ $($ A \& B) represented using alpha-cut principle. Table S4 (supplementary material) lists the Fuzzy Probability of $\mathrm{F}_{\mathrm{M} 1}$, and $\mathrm{F}_{\mathrm{M} 2}$ using $\propto$-cut principle. Upper bound and lower bound in Table 4 are assessed using Equations 9 and 10 , respectively. Whereas, MOM method is used to defuzzify the fuzzy probability of Mitigation Strategies 1 and 2 using Equations 11 and 12, respectively.

$$
\begin{aligned}
& \text { Upper bound }=\mathrm{a}+(\mathrm{b}-\mathrm{a}) \cdot \propto \\
& \text { Upper bound }=\mathrm{d}-(\mathrm{d}-\mathrm{c}) \cdot \propto \\
& \mathrm{F}_{\mathrm{P}} \mathrm{FM}_{1}=\left(\frac{58+70}{2}\right)=64 \% ; \\
& \mathrm{F}_{\mathrm{P}} \mathrm{FM}_{2}=\left(\frac{71+82}{2}\right)=76.5 \% .
\end{aligned}
$$

Fuzzy importance analysis is conducted following the steps described in quantitative FT analysis section to identify level of contribution of each BE to fuzzy probability of each mitigation strategy failure. Table S5 (supplementary material) lists fuzzy importance analysis for failure of Mitigation Strategies 1 and 2.

\subsection{Conducting fuzzy event tree analysis}

After performing quantitative FT analysis for critical risks, and for mitigation strategies failure, fuzzy ET analysis is performed considering the procedure proposed by (Abdelgawad \& Fayek, 2011). Fuzzy arithmetic operation is used on fuzzy numbers to assess EMV using Equation 13 (Verma et al., 2007).

$$
A^{\propto}+B^{\propto}=\left[\mathrm{a}_{1}+\mathrm{a}_{2}, \mathrm{~b}_{1}+\mathrm{b}_{2}, \mathrm{c}_{1}+\mathrm{c}_{2}, \mathrm{~d}_{1}+\mathrm{d}_{2}\right],
$$

where: $\mathrm{A}$ and $\mathrm{B}$ are two trapezoidal fuzzy sets; $A^{\propto}=\left[\mathrm{a}_{1}\right.$, $\left.\mathrm{d}_{1}\right], \mathrm{B}^{\propto}=\left[\mathrm{a}_{2}, \mathrm{~d}_{2}\right]$;

The section of the case study will further explain mathematically how the crisp value of EMV will be obtained as mentioned in the abstract.

\subsection{Case study}

This case considers the situation in Egyptian Real Estate Construction Industry after the Revolution in 2011. The case study is applied to fifteen real estate construction projects. Table S6 (supplementary material) describes the fifteen Real Estate Development projects participated in the case study. This is accomplished through development of Fuzzy Quantitative Risk Analysis model, considering the following stages:

Stage 1: Perform literature review and interviews using indirect method were conducted with fifteen experts. Experts have previous experience of twenty years in both risk assessment and real estate development projects to identify root causes for each critical risk event exists in
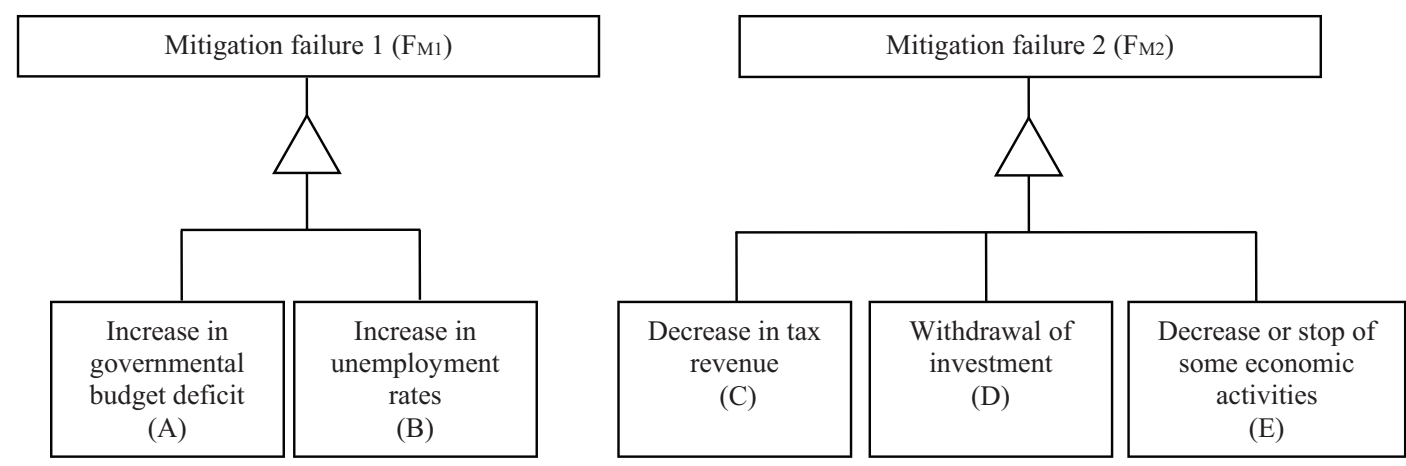

Figure 3. Illustrative example of FT structure 
real estate development project. Also, interviews were conducted to establish linguistic terms to assess occurrence and it was approved with experts to have five linguistic terms which ranges from Very Low (VL) to Very High $(V H)$. Then, the modified horizontal approach with interpolation technique is used to construct MF.

Stage 2: Conduct a qualitative FT Analysis to compute fuzzy probability of BE and to identify MCS.

Stage 3: Conduct a quantitative FT Analysis to assess TE probability.

Stage 4: Perform fuzzy FT analysis for mitigation strategies to determine the failure of selected mitigation strategy to TE.

Stage 5: Perform a Fuzzy ET analysis for each mitigation strategy. After performing a qualitative risk analysis, using Fuzzy Consensus Qualitative Risk Analysis (FCQRA) Framework, twenty-seven risk events were evaluated and ranked. Using the Perateo Principle 20/80, the highest seven critical risk events that were ranked first in the (FCQRA) were introduced to the Fuzzy Quantitative Risk Analysis model. The proposed output is to determine EMV for risk events participated in case study. The case study is then validated through comparing the selling price per square meter before and after $25^{\text {th }}$ January 2011 Revolution to draw a complete picture about the current situation of the Egyptian Real Estate Construction Projects. These critical risks are: 1) Loss due to inflation, 2) Currency devaluation, 3) Increase in borrowing interest rate, 4) Decrease in financially credible contractors' existence, 5) Increase in raw materials prices, 6) Increase in labour expenses, and 7) Increase in equipment costs.

Literature review and interviews using indirect method with fifteen experts are performed to determine causes of each critical risk event using the two step Delphi technique. The process of constructing the membership function was presented elsewhere (Marsh \& Fayek, 2010).
Figure 4 illustrates fault tree structure or the risk event named Loss due to inflation. Equation 14 illustrates the final Minimal Cut Set equation for the Loss due to inflation Risk Event.

$$
\mathrm{TE}=(\mathrm{A} \cup \mathrm{C}) \cup(\mathrm{A} \cap \mathrm{B}) \cup(\mathrm{D} \cap \mathrm{E} \cap \mathrm{F}) .
$$

Experts recommended in interviews using the indirect method and Delphi technique that $F_{P}(A)$ is High, $F_{P}$ $(C)$ is $\operatorname{High}, F_{P}(B)$ is Medium, $F_{P}(D)$ is Medium, $F_{P}(E)$ is Medium, and $F_{P}(F)$ is Low. Fuzzy probability of events is calculated using Equation:

$$
\begin{aligned}
& F_{P}(\mathrm{TE})=1-\left[\left(1-F_{P}(\mathrm{~A})\right) \cdot\left(1-F_{P}(\mathrm{C})\right) \cdot\left(F_{P}(\mathrm{D})\right) \cdot\right. \\
& \left.\left(F_{P}(\mathrm{E})\right) \cdot\left(F_{P}(\mathrm{~F})\right)\right] .
\end{aligned}
$$

Subsequently, Fuzzy FT Analysis is conducted for each mitigation Strategy. Figure 5 illustrates the FT structure of failure of Mitigation Strategy 1 and Mitigation Strategy 2. This is done by performing qualitative fuzzy FT analysis and conducting Fuzzy Quantitative Fault Tree Analysis. Experts recommended that $F_{P}(A)$ is Medium, $F_{P}(B)$ is High, $F_{P}(C)$ is Medium, and $F_{P}(D)$ is High. Equations 16 and 17, represent the failure of Mitigation Strategy 1, whereas, Equations 18 and 19 represent the failure of Mitigation Strategy 1.

$$
\begin{aligned}
& \mathrm{FM}_{1}=\mathrm{A} \cup \mathrm{B} ; \\
& F_{P}\left(\mathrm{FM}_{1}\right)^{\propto}=1-\left(\left(1-F_{P}(\mathrm{~A})^{\propto}\right) \cdot\left(1-F_{P}(\mathrm{~B})^{\propto}\right)\right) ; \\
& \mathrm{FM}_{2}=\mathrm{B} \cup \mathrm{C} \cup \mathrm{D} ; \\
& F_{P}\left(\mathrm{FM}_{2}\right)^{\propto}=1-\left(\left(1-F_{P}(\mathrm{C})^{\propto}\right) \cdot\left(1-F_{P}(\mathrm{D})^{\propto}\right) .\right. \\
& \left.\left(1-F_{P}(\mathrm{E})^{\propto}\right)\right) .
\end{aligned}
$$

Table 1 represents ERM and EMV Calculations for risk "Loss due to Inflation" using mean of maximum method. The EMV equals the value of the middle values of the trapezoidal membership functions divided by 2. Its Expected Monetary Value is calculated using Equation 20.

$$
\mathrm{EMV}=((0.046+0.024) / 2) \cdot 100=3.5 \% \text {. }
$$

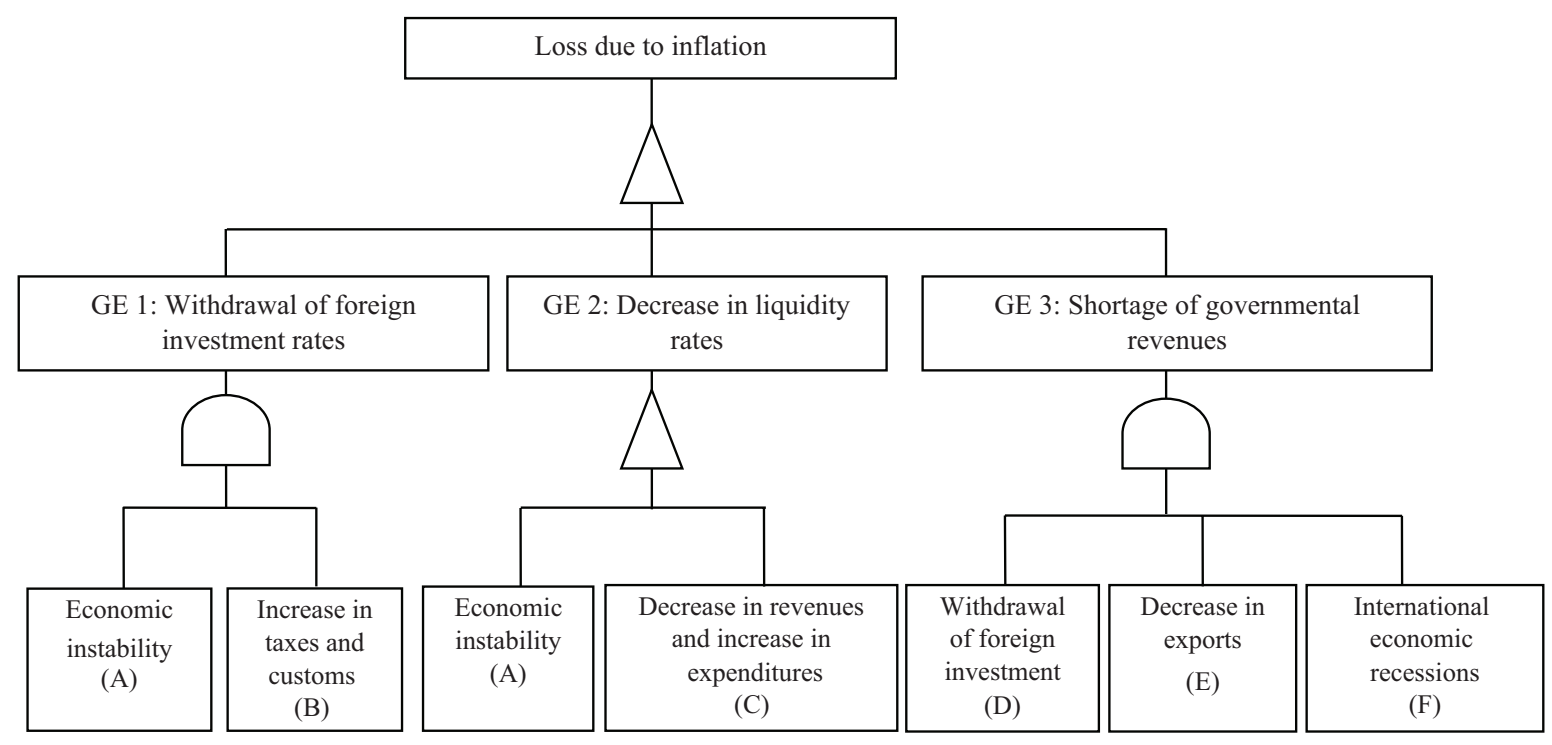

Figure 4. Fault tree structure for risk event "Loss due to inflation" 

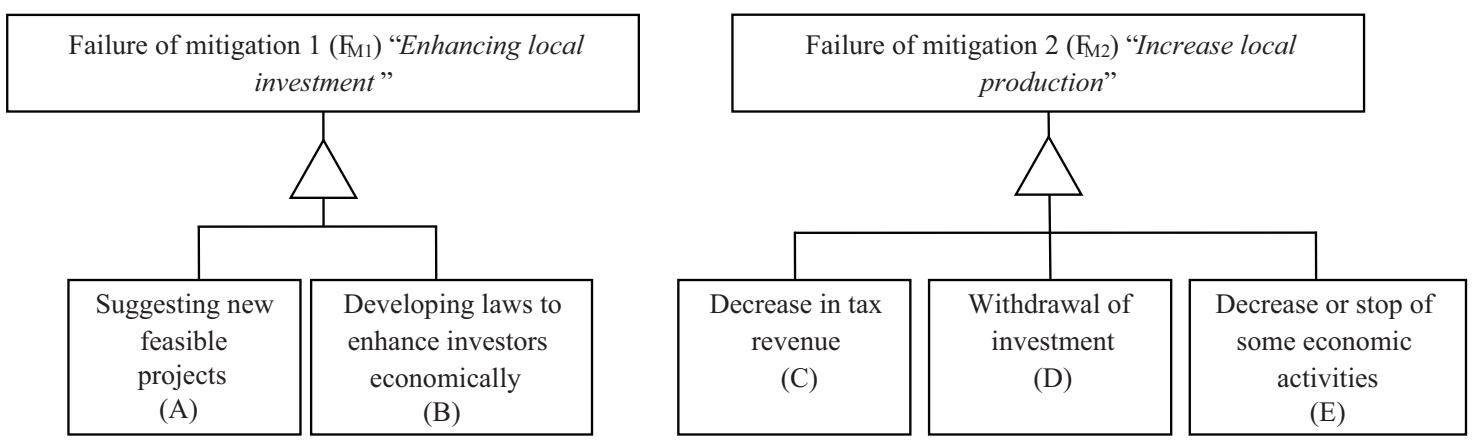

Figure 5. The fault tree structure for each mitigation strategy "Loss due to inflation"

Table 1. Estimated ERM and EMV for "Loss due to inflation" risk event

\begin{tabular}{|c|c|c|c|c|c|c|c|c|c|}
\hline \multirow{2}{*}{ Path } & \multirow{2}{*}{ OP } & \multicolumn{4}{|c|}{ Consequence (C) } & \multicolumn{4}{|c|}{ Expected risk magnitude (ERM) } \\
\cline { 2 - 12 } & & A & B & C & D & A & B & C & D \\
\hline Path1 & 0.072 & 0 & 0 & 0.03 & 0.05 & 0 & 0 & 0.002 & 0.0036 \\
\hline Path2 & 0.287 & 0 & 0.03 & 0.05 & 0.08 & 0 & 0.009 & 0.0144 & 0.023 \\
\hline Path3 & 0.51 & 0 & 0.03 & 0.05 & 0.08 & 0 & 0.0153 & 0.0255 & 0.041 \\
\hline Path4 & 0.127 & 0 & 0 & 0.03 & 0.05 & 0 & 0 & 0.004 & 0.0064 \\
\hline
\end{tabular}

To evaluate the results obtained from case study, a comparative study is conducted. Interviews are held using the indirect method with the fifteen experts whom met before. The allowance of mitigation increased due to the political, economic, and social circumstances that the Egyptian Real Estate Sector faced after 25 ${ }^{\text {th }}$ Jan 2011 Revolution which had strong impacts on the Egyptian Real Estate sector. Fifteen Real Estate Experts provided a selling price for a Real Estate building before and after revolution based on their projects. The Real Estate building is composed of ten apartments. Each apartment has an area of $150 \mathrm{~m}^{2}$. The selling price contains the cost to complete finishes of apartments. After finalizing stages of the Fuzzy Quantitative Risk Analysis Model, EMV was computed for each risk event as percentage of baseline cost. Table 2 lists EMV for each risk, and Fuzzy Probability of Risk Events.

Table 2. EMV for different risk events

\begin{tabular}{|l|c|c|}
\hline \multicolumn{1}{|c|}{ Risk event } & $\begin{array}{c}\text { EMV } \\
\text { percentage }\end{array}$ & $\begin{array}{c}\text { Fuzzy } \\
\text { probability (\%) }\end{array}$ \\
\hline Loss due to inflation & 3.50 & 99.55 \\
\hline Currency devaluation & 4.00 & 100.00 \\
\hline $\begin{array}{l}\text { Increase in borrowing interest } \\
\text { rate }\end{array}$ & 2.05 & 90.80 \\
\hline Increase in labour expenses & 1.51 & 99.25 \\
\hline Increase in equipment costs & 2.55 & 99.20 \\
\hline Increase in raw materials prices & 2.25 & 99.20 \\
\hline $\begin{array}{l}\text { Decrease in financially credible } \\
\text { contractors existence }\end{array}$ & 1.55 & 99.20 \\
\hline Total EMV & 17.41 & \\
\hline
\end{tabular}

To evaluate results obtained from case study, interviews are held using indirect method with fifteen experts. Experts have twenty years of previous experience in both risk assessment, and real estate projects to determine the total construction costs for the Real Estate Building before and after the Egyptian $25^{\text {th }}$ January 2011 Revolution. The Allowances for Mitigation, which increased due to the political, economic, and social circumstances that the Egyptian Real Estate Sector faced after $25^{\text {th }}$ January 2011 Revolution, was calculated by multiplying the percentage of EMV for each risk event with total construction costs for the Real Estate Building price to determine the proposed price of the model. Table 3 lists the calculations of the Expected Monetary Value (EMV), Owners' Profit and the Total Construction Costs of Real Estate Building $/ \mathrm{m}^{2}$ before the $25^{\text {th }}$ January 2011 Egyptian Revolution using proposed model. As illustrated in Table 3, the EMV Value was computed by multiplying the percentage of EMV obtained from Table 2 by the total construction cost per square meter. The proposed Model Price was calculated by adding the total construction costs per square meter, the value of EMV per square meter, and the amount of profit and other real estate expenses per square meter.

Table 4 lists the computations of the Total Construction Costs of Real Estate Building after Revolution $/ \mathrm{m}^{2}$, the Owners' Profit, and the Actual Selling Price of the Real Estate Building per square meter after the $25^{\text {th }}$ Jan Egyptian Revolution. After that percentage error was calculated between the Proposed Price of model per meter square and the Actual Selling Price of the Real Estate Building per meter square after the $25^{\text {th }}$ Jan Egyptian Revolution. Equation (21) describes the percentage error. 
Table 3. Projects' calculated EMV and owners' profit/expenses before $25^{\text {th }}$ January 2011 Egyptian Revolution

\begin{tabular}{|c|c|c|c|c|}
\hline Project ID & $\begin{array}{c}\text { Construction costs } \\
\left(\mathrm{LE} / \mathrm{m}^{2}\right)\end{array}$ & $\begin{array}{c}\text { EMV value } \\
\left(\mathrm{LE} / \mathrm{m}^{2}\right)\end{array}$ & $\begin{array}{c}\text { Owners } \\
\text { profit/expenses } \\
\left(\mathrm{LE} / \mathrm{m}^{2}\right)\end{array}$ & $\begin{array}{c}\text { Model estimated price } \\
\left(\mathrm{LE} / \mathrm{m}^{2}\right)\end{array}$ \\
\hline 1 & $2,944.6$ & 512.7 & 588.9 & 4046 \\
\hline 2 & $3,073.8$ & 535.1 & 461.1 & 3070 \\
\hline 3 & $2,683.1$ & 467.1 & 670.8 & 3975 \\
\hline 4 & $2,893.0$ & 503.7 & 578.6 & 4000 \\
\hline 5 & $2,809.0$ & 489.0 & 702.3 & 4188 \\
\hline 6 & $3,048.0$ & 530.7 & 609.6 & 4215 \\
\hline 7 & $3,183.6$ & 554.3 & 477.5 & 1122 \\
\hline 8 & 880.6 & 153.3 & 88.1 & 1257 \\
\hline 9 & 986.2 & 171.7 & 98.6 & 2552 \\
\hline 10 & $1,941.8$ & 338.1 & 271.8 & 3904 \\
\hline 11 & $2,841.3$ & 494.7 & 568.3 & 898 \\
\hline 12 & 704.5 & 122.7 & 181.1 & 2308 \\
\hline 13 & $1,811.5$ & 315.4 & 547.6 & 3762 \\
\hline 14 & $2,738.0$ & 476.7 & 665.9 & 3793 \\
\hline
\end{tabular}

$\%$ Error $=(($ Proposed Model Price - Actual Selling

Price) / Actual Selling Price) · 100,

where: the Proposed Model Price is the Price obtained from the model for Real Estate Building per square meter, and the Actual Selling Price is the current Price of a Real Estate Building per Square Meter.

Table 5 lists the percentage error between the proposed model estimated price and the actual selling price of a Real Estate Building per square meter for each project due to the political, economic, and social circumstances after the $25^{\text {th }}$ January 2011 Egyptian Revolution.

Table 4. Computations of the actual selling price after $25^{\text {th }}$ January 2011 Egyptian Revolution

\begin{tabular}{|c|c|c|c|}
\hline Project ID & $\begin{array}{c}\text { Total } \\
\text { construction } \\
\left(\mathrm{LE} / \mathrm{m}^{2}\right)\end{array}$ & $\begin{array}{c}\text { Owners' } \\
\text { profit } / \\
\text { expenses } \\
\left(\mathrm{LE} / \mathrm{m}^{2}\right)\end{array}$ & $\begin{array}{c}\text { Actual selling } \\
\left(\mathrm{LE} / \mathrm{m}^{2}\right)\end{array}$ \\
\hline 1 & $3,833.9$ & 575.1 & 4,409 \\
\hline 2 & $4,006.8$ & 360.6 & 4,367 \\
\hline 3 & $3,489.3$ & 558.3 & 4,048 \\
\hline 4 & $3,766.7$ & 489.7 & 4,256 \\
\hline 5 & $3,653.1$ & 548.0 & 4,201 \\
\hline 6 & $3,968.5$ & 595.3 & 4,564 \\
\hline 7 & $4,149.9$ & 373.5 & 4,523 \\
\hline 8 & $1,149.2$ & 57.5 & 1,207 \\
\hline 9 & $1,287.1$ & 64.4 & 1,352 \\
\hline 10 & $2,531.8$ & 227.9 & 2,760 \\
\hline 11 & $3,699.4$ & 554.9 & 4,254 \\
\hline 12 & 919.4 & 46.0 & 965 \\
\hline 13 & $2,364.1$ & 118.2 & 2,482 \\
\hline 14 & $3,564.9$ & 427.8 & 3,993 \\
\hline 15 & $3,464.1$ & 658.2 & 4,122 \\
\hline & & & \\
\hline
\end{tabular}

Table 5. Percentage error of the proposed model

\begin{tabular}{|c|c|c|c|}
\hline Project ID & $\begin{array}{l}\text { Actual selling } \\
\text { price }\left(\mathrm{LE} / \mathrm{m}^{2}\right)\end{array}$ & $\begin{array}{c}\text { Model } \\
\text { estimated } \\
\text { price }\left(\mathrm{LE} / \mathrm{m}^{2}\right)\end{array}$ & Error (\%) \\
\hline 1 & 4409 & 4046 & 8.23 \\
\hline 2 & 4367 & 4070 & 6.8 \\
\hline 3 & 4048 & 3821 & 5.61 \\
\hline 4 & 4256 & 3975 & 6.6 \\
\hline 5 & 4201 & 4000 & 4.78 \\
\hline 6 & 4564 & 4188 & 8.24 \\
\hline 7 & 4523 & 4215 & 6.81 \\
\hline 8 & 1207 & 1122 & 7.04 \\
\hline 9 & 1352 & 1257 & 7.03 \\
\hline 10 & 2760 & 2552 & 7.54 \\
\hline 11 & 4254 & 3904 & 8.23 \\
\hline 12 & 965 & 898 & 6.94 \\
\hline 13 & 2482 & 2308 & 7.01 \\
\hline 14 & 3993 & 3762 & 5.79 \\
\hline 15 & 4122 & 3793 & 7.98 \\
\hline \multicolumn{3}{|c|}{ Average percentage error } & 6.98 \\
\hline
\end{tabular}

As listed in Table 5, the framework had an average percentage error of 6.98, which is acceptable. Experts were then asked to justify why errors existed between the Proposed Price of model and Selling Price of Real Estate Building for the previously mentioned projects. Experts replied that the Expected Monetary Value (EMV) was calculated only for the seven risk events mentioned earlier, while projects contain more risk events, such as Design Fees Increase, and Increase in Governmental Restriction imposed to finance Construction Projects. It is recommended to consider the qualitatively assessed Very High, and High-risk events in the Fuzzy Quantitative Risk 
Analysis Model Framework. After that the results were compared with the previously published works of Abdelgawad (2011), the average percentage error in Abdelgawad (2011) was found to be $13 \%$, while in the proposed model was found to be $6.98 \%$, which gives more reliability in results of proposed model. Based on interviews with fifteen experts in the Egyptian real estate sector, a set of recommendations were made to enhance the Egyptian real estate sector. These recommendations include:

- De-politicize Real Estate system and restructure it into one which reflects market principle.

- Innovative financing models should be offered in foreign currency; for example, mortgages coupled with life insurance.

- Tax incentives should be increased to encourage housing construction.

\section{Conclusions}

A Fuzzy Quantitative Risk Analysis model was developed to quantify risks existed in real estate projects. This was accomplished by using Fault and Event Tree. Risks were identified through the Fuzzy Consensus Measurement Framework, and is limited to independent risk events only. The methodology of developing the fuzzy quantitative risk analysis model involved following five stages; Performing Data collection, Conducting Qualitative FT Analysis, Conducting Quantitative FT Analysis, Analysing Mitigation Strategies Failure, and Conducting Fuzzy ET Analysis. Model is validated and case study was performed using the indirect method and Delphi Technique to validate results obtained from model. Model provided an enhancement over previously published quantitative models by allowing the use of Fuzzy Fault and Event Tree to determine allowance of mitigation of identified risk event. Moreover, the percentage of error was computed between the Proposed Model Price for a real estate building per square meter and the Actual Selling Price for a real estate building per square meter. Furthermore, the average percentage error was computed and validated through comparison with previously published works. The results indicate the reliability of the proposed model since it provides less percentage error.

\section{References}

Abdelgawad, M. (2011). Hybrid decision support system for risk criticality assessment and risk analysis (Thesis dissertation). Faculty of Graduate Studies and Research, Department of Civil and Environmental Engineering, University of Alberta, Edmonton, Alberta, Canada.

Abdelgawad, M., \& Fayek, A. R. (2011). A comprehensive hybrid framework for risk analysis in the construction industry using combined failure mode and effect analysis, fault trees, event trees, and fuzzy logic. Journal of Construction Engineering and Management, 135(5), 642-651. https://doi.org/10.1061/(ASCE)CO.1943-7862.0000471

Abdelhai, M. I. (1993). A new methodology based on fuzzy set theory and fault tree analysis for failure diagnosis in nuclear power plants (Thesis dissertation). Faculty of Graduate Studies and Research, University of Tennessee, USA.

Aboushady, A. (2012). A framework for risk assessment in Egyptian real estate projects using fuzzy approach (M.Sc. Thesis). Cairo University, Cairo, Egypt.

Al-Bahar, J. F., \& Crandall, K. C. (1990). Systematic risk management approach for construction projects. Journal of Construction Engineering and Management, 116(3), 533-546. https://doi.org/10.1061/(ASCE)0733-9364(1990)116:3(533)

Alexandria Bank Economic Research. (2012). Egypt's real estate industry. Working Papers. Retrieved from http://alexbank. smetoolkit.org/egypt/en/file/promotion/ 2552/en/EgyptsRealEstateIndustry2.pdf

Al-Sobiei, O. S., Arditi, D., \& Polat, G. (2005). Predicting the risk of contractor default in Saudi Arabia utilizing artificial neural network (ANN) and genetic algorithm (GA) techniques. Construction Management and Economics, 23(4), 423-430. https://doi.org/10.1080/01446190500041578

Amadeo, K. (2012). How does real estate affect the U.S. economy? US Economy. Retrieved from http://useconomy.about.com/ od/grossdomesticproduct/f/ Real_estate_faq.htm

Angelini, E., Giacomo, T., \& Roli, A. (2008). A neural network approach for credit risk evaluation. Journal of Quarterly Review of Economics and Finance, 48(4), 733-755. https://doi.org/10.1016/j.qref.2007.04.001

Ayyub, B. M. (2003). Risk analysis in engineering and economics (pp. 33-113). Chapter 2. New York: Chapman \& Hall/CRC. https://doi.org/10.1201/9780203497692

Chan, A., Chan, D., \& Yeung, J. (2009). Overview of the application of fuzzy techniques in construction management research. Journal of Construction Engineering and Management, 135(11), 1241-1252.

https://doi.org/10.1061/(ASCE)CO.1943-7862.0000099

Elbarkouky, M., \& Fayek, A. R. (2011). Fuzzy similarity consensus model for early alignment of construction project teams on the extent of their roles and responsibilities. Journal of Construction Engineering and Management, 137(6), 432-441. https://doi.org/10.1061/(ASCE)CO.1943-7862.0000310

Foong, O. M., \& Nordin, M. I. (2010). Decision support system for alarm rationalization using risk assessment matrix. International Journal of Computer Applications, 4(9), 8-13. https://doi.org/10.5120/857-1174

Hauptmanns, U. (1988). Fault tree analysis for process plants. In A. Kandel \& E. Avni (Eds.), Engineering risk and hazard assessment, Vol. I. Boca Raton: CRC Press.

Javid, M., \& Seneviratne, P. N. (2000). Investment risk analysis in airport parking facility development. Journal of Construction Engineering and Management, 126(4), 298-305. https://doi.org/10.1061/(ASCE)0733-9364(2000)126:4(298)

Kangari, R., \& Riggs, L. S. (1989). Construction risk assessment by linguistics. Transactions on Engineering Management IEEE, 36(2), 126-131. https://doi.org/10.1109/17.18829

KarimiAzari, A., Mousavi, N., Mousavi, S. F., \& Hosseini, S. B. (2011). Risk assessment model selection in construction industry. Expert Systems with Applications, 38(8), 9105-9111. https://doi.org/10.1016/j.eswa.2010.12.110

Khan, F., \& Abbasi, S. A. (1999). PROFAT: a user-friendly system for probabilistic fault tree analysis. Process Safety Progress, 18(1), 42-49. https://doi.org/10.1002/prs.680180109

Levner, E., Ganoulis, J., Linlov, I., \& Benayahu, Y. (2007). Multi objective risk/cost analysis of artificial marine systems using decision trees and fuzzy expert estimations. In I. Linkov, G. A. Kiker, \& R. J. Wenning (Eds.), Environmental security in harbors and coastal areas (pp. 161-174). NATO Security through 
Science Series (Series C: Environmental Security). Dordrecht: Springer. https://doi.org/10.1007/978-1-4020-5802-8_12

Maria-Sanchez, P. (2005). Neural-risk assessment system for construction projects. Construction Research Congress, ASCE, San Diego, California, US.

Markowski, A. S., \& Mannan, M. S. (2008). Fuzzy risk matrix. Journal of Hazardous Materials, 159(1), 152-157. https://doi.org/10.1016/j.jhazmat.2008.03.055

Markowski, A. S., Mannan, M. S., \& Bigoszewska, A. (2009). Logic for process safety analysis. Journal of Loss Prevention in the Process Industries, 22(6), 695-702. https://doi.org/10.1016/j.jlp.2008.11.011

Marsh, K., \& Fayek, A. R. (2010). Fuzzy expert system to assist surety underwriters in evaluating construction contractors for bonding. Journal of Construction Engineering and Management, 136(11), 1219-1235. https://doi.org/10.1061/(ASCE)CO.1943-7862.0000224

NASA. (2002). Fault tree handbook with aerospace applications. NASA Office of Safety and Mission Assurance, NASA Headquarters, Washington, USA.

PMI. (2008). A guide to the project management body of knowledge $(P M B O K)\left(4^{\text {th }}\right.$ ed.). The Project Management Institute (PMI), Pennsylvania, USA.

Robert, S. C. (2004, 29-31 March). 3D model for qualitative risk assessment. In SPE International Conference on Health, Safety, and Environment in Oil and Gas Exploration and Production. Calgary, Alberta, Canada.

Sadeghi, N., Fayek, A. R., \& Pedrycz, W. (2010). Fuzzy Monte Carlo simulation and risk assessment in construction. Computer-Aided Civil and Infrastructure Engineering, 25(4), 238252. https://doi.org/10.1111/j.1467-8667.2009.00632.x
Shaheen, A. A., Fayek, A. R., \& AbouRizk, S. M. (2007). Fuzzy numbers in cost range estimating. Journal of Construction Engineering and Management, 133(4), 325-334. https://doi.org/10.1061/(ASCE)0733-9364(2007)133:4(325)

Song, H., Zhang, H., \& Chan, C. (2009). Fuzzy fault tree analysis based on T-S model with application to INS/GPS navigation system. Journal of Soft Computing, 13(1), 31-40. https://doi.org/10.1007/s00500-008-0290-3

Tyagi, S. K., Pandey, D., \& Kumar, V. (2011). Fuzzy fault tree analysis for fault diagnosis of cannula fault in power transformer. Journal of Applied Mathematics, 2(11), 1346-1355. https://doi.org/10.4236/am.2011.211188

Verma, A. K., Srividya, A., \& Gaonkar, R. S. P. (2007). Fuzzyreliability engineering: concepts and applications (pp. 88-127). Chapter 4. New Delhi: Narosa Publishing House.

Wu, J., Yan, S., \& Xie, L. (2011). Reliability analysis method of a solar array by using fault tree analysis and fuzzy reasoning Petri net. Acta Astronautica, 69(11-12), 960-968. https://doi.org/10.1016/j.actaastro.2011.07.012

Yiu, T. W., Cheung, S. O., \& Lok, C. L. (2015). A fuzzy fault tree framework of construction dispute negotiation failure. IEEE Transactions on Engineering Management, 62(2), 171-183. https://doi.org/10.1109/TEM.2015.2407369

Yuhua, D., \& Datao, Y. (2005). Estimation of failure probability of oil and gas transmission pipelines by fuzzy fault tree analysis. Journal of Loss Prevention in the Process Industries, 18(2), 83-88. https://doi.org/10.1016/j.jlp.2004.12.003

Zadeh, L. A. (1965). Fuzzy sets. Information and Control, 8(3), 338-353. https://doi.org/10.1016/S0019-9958(65)90241-X 